\title{
Intestinal carcinogenesis: IKK can go all the way
}

\author{
Yoshiaki Sunami and Thomas Wirth
}

Institute of Physiological Chemistry, University of UIm, UIm, Germany.

\begin{abstract}
Chronic inflammation has long been suspected to support tumorigenesis in a variety of cancers. The I $\kappa B$ kinase (IKK)/NF- $\kappa B$ pathway is the critical signal transduction pathway regulating inflammation, and loss-of-function studies have demonstrated its involvement in tumorigenesis. In this issue of the JCI, Vlantis et al. present evidence that persistent genetic activation of IKK/NF- $K B$ signaling in intestinal epithelial cells not only accelerates tumorigenesis in models of both carcinogen- and mutation-induced colorectal cancer, but also is sufficient to induce intestinal tumors.
\end{abstract}

Inflammation and colorectal cancer

Colorectal cancer is one of the most common malignancies and causes of death worldwide. Each year, more than 1.2 million new cases are registered, and it is estimated that approximately 600,000 people die from the disease (1). The etiological factors for colorectal cancer include genetic mutations, chronic inflammation, diet, and lifestyle (2). Among the dietary and lifestyle risk factors that have been defined are a diet rich in unsaturated fats and red meat, consumption of alcohol, and reduced physical activity (2), all of which are associated with the higher incidence of colorectal cancer in developed countries. Critical mutations underlying the pathogenesis of colorectal cancer have been identified by molecular genetic studies. The main targets of genetic change are the oncogene KRAS and several tumor suppressor genes, most prominently adenomatous polyposis coli (APC) and P53 (2, 3).

Many observations now indicate that chronic inflammation is important in the pathogenesis of colorectal cancer $(2,4)$. For example, inflammatory cells are often found infiltrated in human tumors and preneoplastic lesions (5). These cells produce cytokines and chemokines involved in propagation of the inflammatory response and support the growth and survival of premalignant cells (4). In addition, one of the best established rodent models of intestinal carcinogenesis, the azoxymethane/dextran sulfate sodium model (AOM/DSS model), involves the use of a chronic inflammatory stimulant (DSS), which is critically

Conflict of interest: The authors have declared that no conflict of interest exists.

Citation for this article: J Clin Invest. doi:10.1172/ JCI58454. required for the onset of tumorigenesis. Furthermore, several mouse lines have been generated in which the inflammatory response has been blunted or enhanced through genetic modification. These mice are models for intestinal cancer and provide clear evidence of the link between chronic inflammation and intestinal cancer.

The IкB kinase (IKK)/NF- $\kappa$ B signaling pathway represents the dominant regulator of the inflammatory response. Multiple proinflammatory mediators, including TNF- $\alpha$, trigger activation of the IKK complex, which is made up of the two kinases IKK1 and IKK2 and the regulatory protein NEMO. This results in the phosphorylation and subsequent degradation of IкB proteins, which are the direct negative regulators of NF- $\mathrm{\kappa B}$ transcription factors (Figure 1). In this issue of the JCI, Vlantis et al. directly prove that activation of NF- $\mathrm{KB}$ signaling by overexpression of the activating kinase IKK2 in intestinal epithelial cells (IECs) results in both inflammation and tumorigenesis (6). These data demonstrate

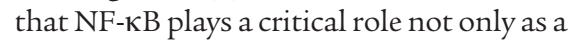
tumor promoter but also as an initiator of the complex process of tumorigenesis.

\section{NF-KB in IECs, a central regulator in tumorigenesis}

To understand the epithelial-intrinsic role of NF- $\mathrm{KB}$ in intestinal tumorigenesis in vivo, Vlantis and colleagues (6) generated mice expressing a constitutively active Ikk2 allele (7) in IECs, utilizing mice that express Cre under the control of the villin promoter (8). Villin is an actin-binding protein expressed in the brush border of the gut epithelium. The consequence of constitutive expression of the active allele of Ikk2 in IECs was that NF-кB became activated in these cells (6). These mice exhibited mild intestinal inflammation and immune cell infiltration, mainly by macrophages and granulocytes. IKK2-expressing IECs showed upregulation of proinflammatory cytokines, including TNF- $\alpha$ and IL- $1 \beta$, and chemokines, which mediate the infiltration of immune cells in a paracrine manner. Of particular interest in the context of colorectal cancer, the mice developed more severe colitis when treated with DSS alone, accelerated tumorigenesis in the AOM/DSS model of colorectal cancer, and even epithelial hyperplasia and early dysplastic lesions in the intestine after repeated injection of only AOM. Furthermore, when these mice were crossed with mice bearing a truncated Apc allele, a strong enhancement of tumor formation was observed. Most strikingly, activation of the IKK/NF-кB pathway in IECs was sufficient to induce intestinal tumors in aged mice after a latency period of ongoing chronic inflammation. With respect to the mechanism by which IKK/NF-KB signaling accelerated/induced intestinal tumors, both cell-intrinsic and paracrine factors were identified. Enhanced expression of cytokines and chemokines providing a tumor-promoting proinflammatory microenvironment was detected, as was induction of the $\mathrm{Wnt} / \beta$-catenin pathway. This might explain the switch from inflammation to transformation observed by Vlantis and colleagues, because activation of the $\mathrm{Wnt} / \beta$-catenin pathway is widely accepted as the critical mechanism in intestinal carcinogenesis (3). Taken together, the findings of Vlantis and colleagues firmly

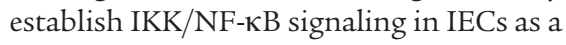
master regulator for both intestinal inflammation and tumorigenesis.

\section{NF-KB and inflammation in intestinal tumorigenesis revisited}

NF- $\kappa \mathrm{B}$ regulates DSS-induced tumorigenesis. A variety of genetic models have been analyzed that support this conclusion. These models have mainly studied the consequences of blocked proinflammatory signaling. Deletion of IKK2 in IECs decreases the incidence of AOM/DSS-induced colitis-associated cancer, but does not prevent DSS-induced inflammation (9). The reduced 


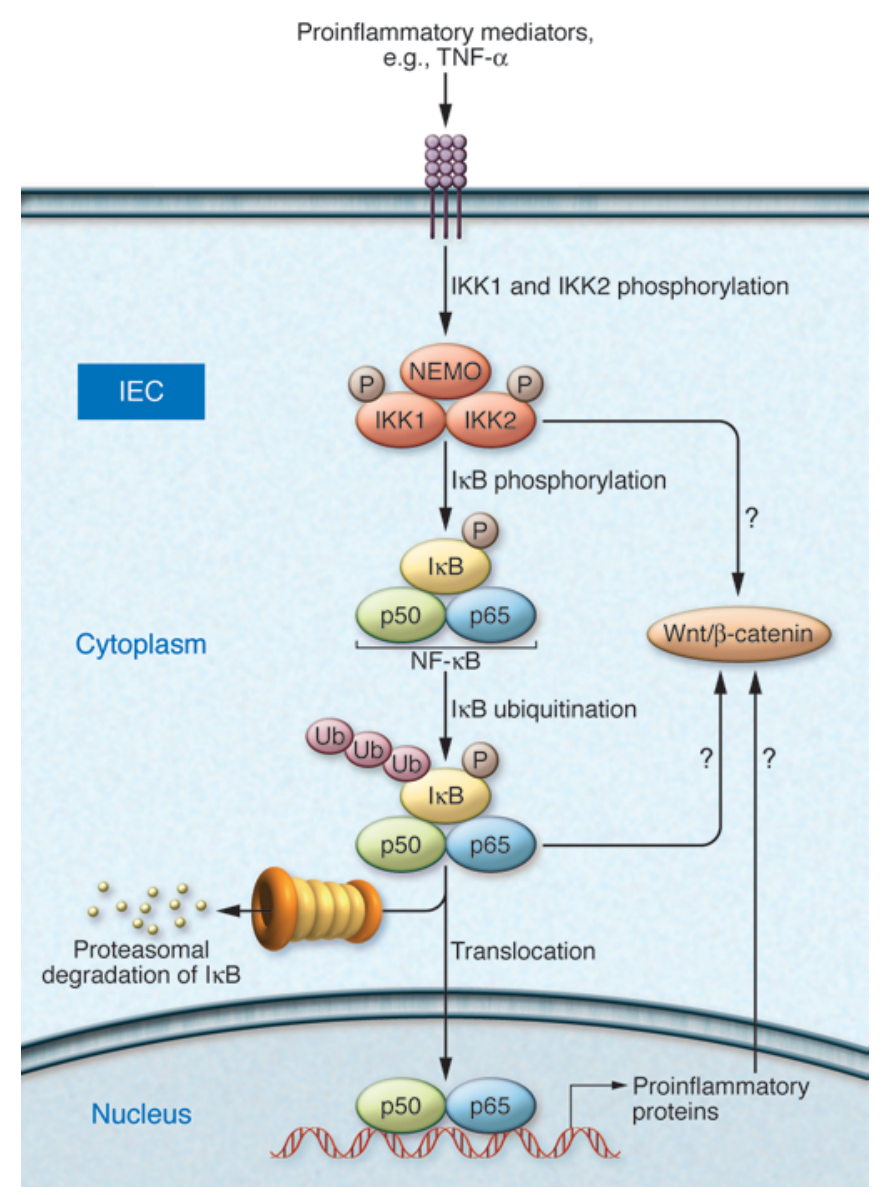

tumor incidence in these mice is consistent with the tumor-promoting function of IKK2 in IECs shown by Vlantis et al. (6). In line with these results, mice in which TNF- $\alpha$ signaling, which is mediated via the IKK2/ NF-кB pathway, is blocked show reduced mucosal damage, immune cell infiltration, and colitis-associated tumorigenesis following administration of AOM/DSS (10).

Cross talk between intestinal inflammation and NF- $\mathrm{KB}$ has been proposed previously following a series of genetic experiments (11). Mice in which either NEMO, the regulatory molecule critical for activation of NF-אB signaling, or both IKK1 and IKK2 are specifically deleted in IECs develop spontaneous severe chronic intestinal inflammation but no tumors. This is because NF- $\mathrm{KB}$ deficiency in IECs leads to enterocyte apoptosis via TNF- $\alpha$, which results in disruption of gut barrier function and concurrently drives chronic inflammation by cytokines and chemokines produced by infiltrated immune cells fighting the resulting invading bacteria (11). Taken together with the current data of Vlantis et al. (6), these results indicate that both activation and inactiva- tion of the NF-אB pathway in IECs can lead to intestinal inflammation, but the underlying mechanisms are quite distinct.

The role of NF-кB in intestinal inflammation and cancer has also been addressed in mouse models modulating negative regulators of the NF-кB pathway. Mice with IEC-specific deletion of A20, an NF-кB target gene that is essential for the termination of NF- $\kappa \mathrm{B}$ activation, are sensitized for DSS-induced colitis (12). In addition, mice lacking the cylindromatosis (Cyld) gene, which encodes a negative regulator of the NF- $\kappa \mathrm{B}$ activation pathway, exhibit increased chronic intestinal inflammation and susceptibility to colitis-associated cancer (13). These observations further support the conclusion that dysregulation of NF- $\kappa \mathrm{B}$ in IECs supports inflammation and colitis-associated cancer.

\section{NF-KB regulates subordinate activation of Wnt/B-catenin}

Mutations of the APC tumor suppressor gene lead to familial adenomatous polyposis (FAP), an autosomal dominant hereditary disease that is one of the principle pre-

\section{Figure 1}

IKK/NF-KB signaling as a key mediator of intestinal inflammation and tumorigenesis. Proinflammatory cytokines, such as TNF- $\alpha$, trigger phosphorylation and activation of the IKK complex, which is composed of two catalytic kinase subunits (IKK1 and IKK2) and the regulatory subunit NEMO. As a consequence, the inhibitory protein I $\mathrm{KB}$ is phosphorylated, ubiquitinated, and then degraded by the proteasome. The NF-kB dimer (e.g., p50 and p65) translocates into the nucleus and regulates target gene expression. Vlantis et al. overexpressed constitutively active IKK2 in IECs (6). This led to inflammation (immune cell infiltration) and tumorigenesis via intrinsic and paracrine mechanisms. As depicted here, they found that overexpression of constitutively active IKK2 led to activation of the Wnt/ $\beta$-catenin pathway. However, it is not yet clear how this is regulated (directly via IKK or

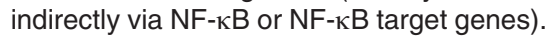

dispositions to colorectal cancer $(14,15)$. The so-called Apc1638N mouse carries a targeted mutant allele at the endogenous Apc gene and spontaneously develops adenomas and adenocarcinomas of the small intestine at approximately 6 months of age (16). APC is known to downregulate $\beta$-catenin activity, and inactivation of APC leads to stabilization of $\beta$-catenin (17). There are several known downstream targets of APC $/ \beta$-catenin, including MYC and cyclin D1, which are considered highly relevant in tumor formation (2). In their study, Vlantis et al. showed that activation of the NF-KB pathway in IECs through overexpression of constitutively active IKK2 accelerates tumorigenesis in Apc1638N mice (6). They also showed increased $\beta$-catenin activity in IKK2-expressing IECs. However, they did not observe induction of $M y c$ and cyclin D1 in Apc1638N mice after activation

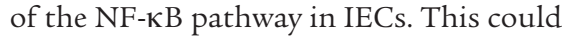
be due to upregulation of SOX9, a Wnt/ $\beta$-catenin target (18), which can repress Myc and cyclin D1 expression. Overlapping as well as distinct mechanisms of tumorigenesis supported by Wnt/ $\beta$-catenin sig- 
naling and NF-кB signaling might exist. In any case, the new work by Vlantis et al. (6) demonstrates that constitutive IKK2 activity results in activation of the Wnt/ $\beta$-catenin pathway. However, it remains an open question whether IKK2 directly activates $\mathrm{Wnt} / \beta$-catenin or indirectly activates Wnt $/ \beta$-catenin via NF- $\kappa \mathrm{B}$ or NF- $\mathrm{NB}$ target genes, even in a paracrine fashion.

\section{Relevance to human colorectal cancer?}

What is the relevance of the findings of Vlantis et al. (6) for colorectal cancer in humans? Activating mutations in intrinsic components of the IKK/NF-кB pathway are not commonly found in human colorectal cancer, but patients suffering from chronic intestinal inflammation (inflammatory bowel disease) do have an increased risk of developing intestinal tumors. The genetic model used by Vlantis et al. relied on the specific overexpression of a constitutively active allele of Ikk2; the other components of the IKK complex (IKK1 and NEMO) were not correspondingly induced. It is therefore possible that this selective expression unleashed a function of IKK2, potentially beyond its prime target IKB, that is better controlled when IKK2 is in its physiological complex. Nevertheless, the data impressively demonstrate that IKK2 in the absence of any additional carcinogen, via intrinsic (poten- tially Wnt/ $\beta$-catenin) as well as paracrine (tumor-supporting inflammatory cytokine milieu) mechanisms, induces inflammation, stem cell proliferation, and finally neoplastic transformation of IECs (6). Its targeting might well represent an attractive therapeutic option in colorectal cancer.

\section{Acknowledgments}

The authors' work is in part supported by German Research Foundation grant SFB 518 TP B23.

Address correspondence to: Thomas Wirth, Institute of Physiological Chemistry, University of Ulm, Albert-Einstein-Allee 11, 89081 Ulm, Germany. Phone: 49.731.500.23270; Fax: 49.731.500.22892; E-mail: thomas. wirth@uni-ulm.de.

1. International Agency for Research on Cancer. GLOBOCAN Web site. http://globocan.iarc.fr/. Updated December 2010. Accessed April 29, 2011.

2. Fearon ER. Molecular genetics of colorectal cancer. Annu Rev Pathol. 2011;6:479-507.

3. Fodde R, Smits R, Clevers H. APC, signal transduction and genetic instability in colorectal cancer. Nat Rev Cancer. 2001;1(1):55-67.

4. Karin M, Lawrence T, Nizet V. Innate immunity gone awry: linking microbial infections to chronic inflammation and cancer. Cell. 2006;124(4):823-835.

5. Coussens LM, Werb Z. Inflammation and cancer. Nature. 2002;420(6917):860-867.

6. Vlantis K, et al. Constitutive IKK2 activation in intestinal epithelial cells induces intestinal tumors in mice. J Clin Invest. 2011;121(7):2781-2793.

7. Sasaki Y, et al. Canonical NF-kappaB activity, dis- pensable for B cell development, replaces BAFFreceptor signals and promotes $B$ cell proliferation upon activation. Immunity. 2006;24(6):729-739.

8. Madison BB, Dunbar L, Qiao X T, Braunstein K, Braunstein E, Gumucio DL. Cis elements of the villin gene control expression in restricted domains of the vertical (crypt) and horizontal (duodenum, cecum) axes of the intestine. J Biol Chem. 2002; 277(36):33275-33283.

9. Greten FR, et al. IKKbeta links inflammation and tumorigenesis in a mouse model of colitis-associated cancer. Cell. 2004;118(3):285-296.

10. Popivanova BK, et al. Blocking TNF-alpha in mice reduces colorectal carcinogenesis associated with chronic colitis. J Clin Invest. 2008;118(2):560-570.

11. Nenci A, et al. Epithelial NEMO links innate immunity to chronic intestinal inflammation. Nature. 2007;446(7135):557-561.

12. Vereecke L, et al. Enterocyte-specific A20 deficiency sensitizes to tumor necrosis factor-induced toxicity and experimental colitis. J Exp Med. 2010; 207(7):1513-1523.

13. Zhang J, et al. Impaired regulation of NF- $\kappa B$ and increased susceptibility to colitis-associated tumorigenesis in CYLD-deficient mice. J Clin Invest. 2006;116(11):3042-3049.

14. Miyoshi Y, et al. Somatic mutations of the APC gene in colorectal tumors: mutation cluster region in the APC gene. Hum Mol Genet. 1992;1(4):229-233.

15. Su LK, et al. Multiple intestinal neoplasia caused by a mutation in the murine homolog of the APC gene. Science. 1992;256(5057):668-670.

16. Smits R, et al. Loss of Apc and the entire chromosome 18 but absence of mutations at the Ras and Tp53 genes in intestinal tumors from Apc1638N, a mouse model for Apc-driven carcinogenesis. Carcinogenesis. 1997;18(2):321-327.

17. Morin PJ, et al. Activation of beta-catenin-Tcf signaling in colon cancer by mutations in beta-catenin or APC. Science. 1997;275(5307):1787-1790.

18. Bastide P, et al. Sox9 regulates cell proliferation and is required for Paneth cell differentiation in the intestinal epithelium. J Cell Biol. 2007;178(4):635-648. 